\title{
Software Engineering
}

National Cancer Institute

\section{Source}

National Cancer Institute. Software Engineering. NCI Thesaurus. Code C17147.

The application of a systematic, disciplined, quantifiable approach to the development, operation, and maintenance of computer software. 